\title{
Zoledronic acid impairs stromal reactivity by inhibiting M2-macrophages polarization and prostate cancer-associated fibroblasts
}

\author{
Giuseppina Comito ${ }^{1}$, Coral Pons Segura ${ }^{1}$, Maria Letizia Taddei ${ }^{1}$, Michele Lanciotti ${ }^{3}$, \\ Sergio Serni ${ }^{3}$, Andrea Morandi ${ }^{1}$, Paola Chiarugi ${ }^{1,2}$, Elisa Giannoni ${ }^{1}$ \\ ${ }^{1}$ Department of Experimental and Clinical Biomedical Sciences, University of Florence, 50134 Florence, Italy \\ ${ }^{2}$ Tuscany Tumor Institute and "Center for Research, Transfer and High Education DenoTHE", 50134 Florence, Italy \\ ${ }^{3}$ Department of Urology, University of Florence, Careggi Hospital, Urologic Clinic San Luca, 50100 Florence, Italy \\ Correspondence to: Elisa Giannoni, email: elisa.giannoni@unifi.it \\ Keywords: zoledronic acid, prostate cancer, cancer-associated fibroblasts, macrophages, mevalonate pathway \\ Received: January 29, 2016 Accepted: May 05, $2016 \quad$ Published: May 20, 2016
}

\section{ABSTRACT}

Zoledronic acid (ZA) is a biphosphonate used for osteoporosis treatment and also proved to be effective to reduce the pain induced by bone metastases when used as adjuvant therapy in solid cancers. However, it has been recently proposed that ZA could have direct anti-tumour effects, although the molecular mechanism is unknown. We herein unravel a novel anti-tumour activity of ZA in prostate cancer ( $\mathrm{PCa}$ ), by targeting the pro-tumorigenic properties of both stromal and immune cells. Particularly, we demonstrate that ZA impairs PCa-induced M2-macrophages polarization, reducing their pro-invasive effect on tumour cells and their pro-angiogenic features. Crucially, ZA administration reverts cancer associated fibroblasts (CAFs) activation by targeting the mevalonate pathway and RhoA geranyl-geranylation, thereby impairing smooth muscle actin- $\alpha$ fibers organization, a prerequisite of fibroblast activation. Moreover, ZA prevents the M2 macrophages-mediated activation of normal fibroblast, highlighting the broad efficacy of this drug on tumour microenvironment. These results are confirmed in a metastatic xenograft PCa mouse model in which ZA-induced stromal normalization impairs cancer-stromal cells crosstalk, resulting in a significant reduction of primary tumour growth and metastases. Overall these findings reinforce the efficacy of ZA as a potential therapeutic approach to reduce cancer aggressiveness, by abrogating the supportive role of tumour microenvironment.

\section{INTRODUCTION}

Inflammation is now acknowledged as a hallmark of cancer and several tumour-associated cells are active players in promoting a pro-inflammatory microenvironment, including cancer associated fibroblasts (CAFs) and tumour-associated macrophages (TAMs). These accessory cells establish a malignant cross-talk with cancer cells that affects the behaviour of each other, ultimately allowing cancer to acquire aggressive features [1].

CAFs, one of the major components of tumour microenvironment, have been reported to induce epithelial-to-mesenchymal transition (EMT), invasiveness, resistance to anoikis and chemotherapeutic agents, as well as stem-like features of cancer cells, thereby contributing to metastatic spread [2-4]. CAFs also cooperate with tumour cells in fostering de novo angiogenesis [5]. In addition, CAFs establish a metabolic cross-talk with cancer cells, an acknowledgment prerequisite of highly aggressive tumours. Particularly, prostate cancer (PCa) cells meet their energetic and anabolic requirements by exploiting the lactate produced by glycolysis-dependent CAFs [6, 7].

CAFs have also been reported to induce the establishment of a pro-inflammatory microenvironment, by concurring to monocyte recruitment to tumour site and polarization into the pro-tumoral subset of M2 macrophages in a stromal derived growth factor-1dependent manner [8]. In turn, M2 macrophages support malignant progression by promoting angiogenesis and the establishment of an immunosoppressive microenvironment 
$[9,10]$. In keeping, switch of M1 macrophages, endowed with immune stimulating and anti-tumour activities, towards the M2 phenotype is associated with a worse clinical prognosis and high grade of malignancy $[11,12]$. Recent studies also demonstrate that M2 macrophages are able to modulate stromal reactivity, by inducing fibroblast activation [8]. This interplay between CAFs and M2 macrophages promotes (i) tumour cell motility, hence favouring cancer cells to leave the primary tumour site and (ii) the activation of both endothelial cells and their bonemarrow-derived precursors to drive de novo angiogenesis, a step that facilitate metastatic dissemination [8].

Zoledronic acid (ZA) is a FDA-approved thirdgeneration amino-bisphosphonate that has been used as adjuvant therapy to reduce skeletal related events (SREs) and pain associated with bone metastases in several types of cancer, including PCa [13]. Additionally, $\mathrm{ZA}$ has been found to have a direct anti-tumour efficacy. Indeed, ZA inhibits cell proliferation and induces apoptosis in human leukemic cell lines [14]. In addition, ZA directly target endothelial progenitor cells interfering with their differentiation, thus impairing their supportive role in cancer cells escape from primary sites [15]. More recently, ZA has been shown to improve immunesurveillance against tumour and to modulate macrophage differentiation, opening new possibilities for its therapeutic application [16]. However, the molecular mechanisms behind the anti-cancer response of ZA and its effects on non-cancerous stromal cells, remains yet largely unclear and need to be clarified.

Here, we report that in PCa cellular models ZA impairs both M2 macrophages differentiation and CAF activation, thus impairing their supportive roles in $\mathrm{PCa}$ tumour progression. This suggests that the benefit of ZA in the therapy of PCa-bearing patients, potentially goes beyond the simple skeletal/bone symptoms treatment, but is enlarged to regulation of stromal inflammatory events, with a consequent impact on cancer cells malignancy.

\section{RESULTS}

\section{ZA treatment impairs M2 macrophages polarization}

We have recently reported that $\mathrm{PCa}$ cells recruit monocytes and polarize them towards an M2 macrophages phenotype, which in turn promote cancer cell invasiveness [8]. Since ZA has been proposed to have anti-tumoral and immunomodulatory properties [17], we aim at investigating the effect of the compound on this feed-forward loop between cancer and immune cells. Monocytes isolated from normal human blood donors buffy coat were treated with macrophage colony-stimulating factor (MCSF) to promote differentiation into macrophage, enabling them to proliferate and later acquire a specific polarization into M1, M2 or M2-like macrophages upon stimulation with LPS+IFN- $\gamma$, IL-4 or CM from PC3, respectively. Of note, treatment to polarize towards a M1, M2 or M2like phenotype by means of specific treatment During the differentiation period, ZA was administrated at different doses (Supplementary Figure S1A).

To analyse if ZA treatment can impact on macrophages polarization, we evaluated the production of IL-10 and IL-12. Indeed, while M1 macrophages are characterized by $\mathrm{IL}-12^{\text {high }} / \mathrm{IL}-10^{\text {low }}$ phenotype, M2 macrophages show an IL-12 $2^{\text {low }} / \mathrm{IL}-10^{\text {high }}$ production [18]. The results demonstrate that treatment with ZA reduces IL10 expression without affecting IL-12 levels, suggesting a drug-dependent impairment of M2 polarization, without a conversion towards the M1 phenotype (Figure 1A1B). Notably, ZA appears to be effective at the lower dose of $200 \mathrm{nM}$, without any significant dose-dependent effect. To further analyse the M1/M2 subtype modulation upon ZA administration, we analysed the expression of MCSF receptor (MCSF-R), in order to confirm the acquisition of the differentiated phenotype, CD206 as a cell surface marker for M2 polarized macrophages and cycloxigenase-2 (COX-2) and iNOS as markers for the M1 subtype. MCSF-R is highly expressed by macrophages cultured with MCSF, as well as by macrophages incubated with CM from PC3 or treated with ZA. In contrast, only M1 macrophages express high level of COX-2 (Figure 1C). These data highlight that ZA can reduce the pool of pro-tumoral M2-polarized macrophages, without promoting a real unbalance towards the anti-tumoral M1polarized macrophages.

\section{ZA-treated M2-polarized macrophages negatively affect invasiveness of PCa cells}

We have reported that M2 and M2-like macrophages are able to increase cancer cells malignancy [8]. To evaluate the effects of both M2 or M2-like macrophages treated with ZA on PC3 cells motility, CM from the above-treated macrophages were collected and administered to PCa cells for $24 \mathrm{~h}$. An invasion assay was then performed. In agreement with our previous data, M2 and M2-like macrophages elicit a pro-invasive effect on PC3 cells, which is significantly impaired by ZA administration (Figure 2A-2B). Importantly, PC3 cell survival was unaffected by ZA treatment, therefore excluding an effect on cell viability rather than on cell motility (Supplementary Figure S2). These data reveals that ZA-dependent inhibition of M2 polarization leads to an indirect impairment of tumour cell invasion.

\section{ZA hinders the angiogenic response induced by M2 and M2-like macrophages}

We demonstrated that M2 macrophages contribute to increase tube-like structures formation in EPCs and HUVECs, suggesting a key role of immune cells 
A

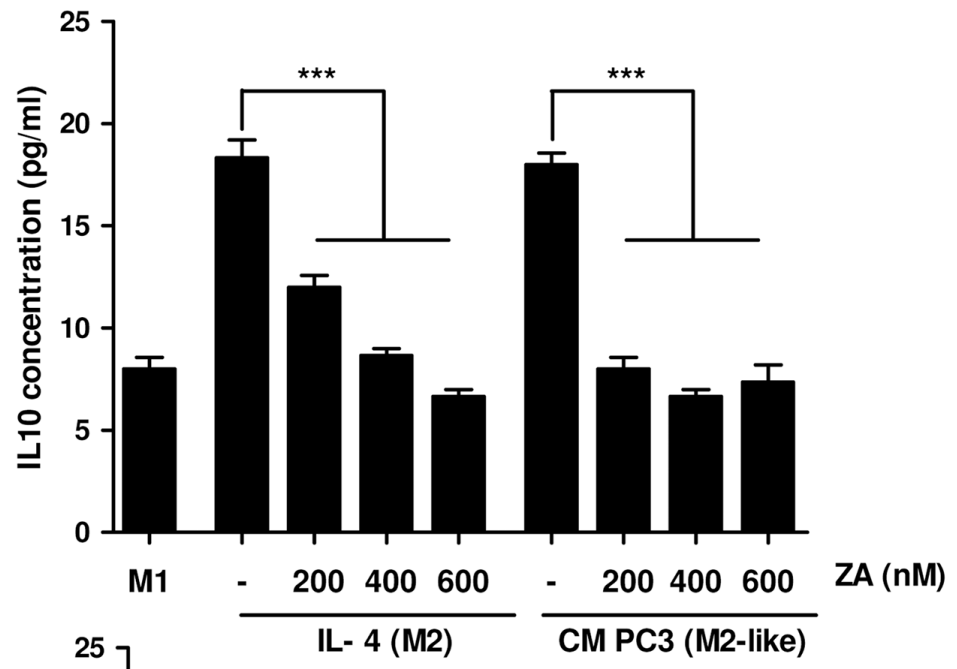

B

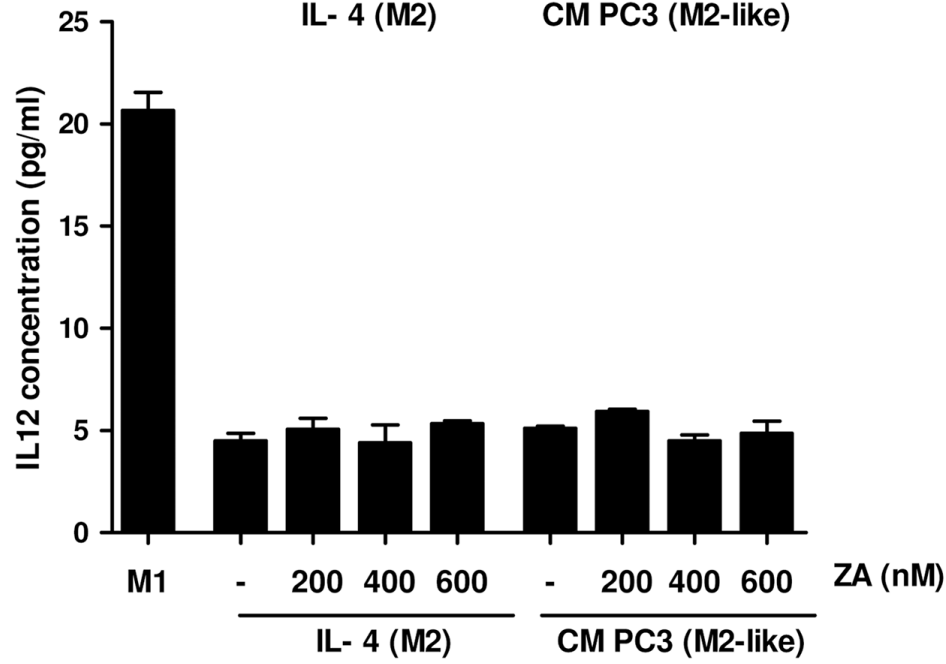

C

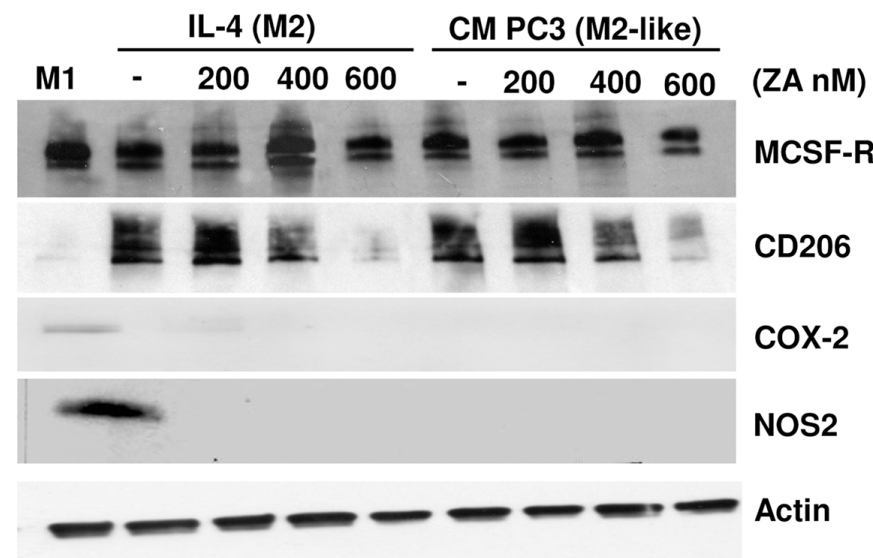

Figure 1: ZA suppresses monocyte differentiation toward M2 macrophages A, B. Human monocytes isolated from normal donor buffy coat were cultured for 7 days with M-CSF $(50 \mathrm{ng} / \mathrm{ml})$. Differentiation toward the M1 phenotype was induced by treatment with LPS $(100 \mathrm{ng} / \mathrm{ml})$ and IFN $\gamma(100 \mathrm{ng} / \mathrm{ml})$ for $24 \mathrm{~h}$. M2 macrophages were polarized by stimulating with IL-4 (20 ng/ml) for $24 \mathrm{~h}$. Alternatively, M2-like macrophages were obtained by treating isolated monocytes with CM from PC3 for 7 days. During differentiation macrophages were treated with different concentrations of ZA and the levels of IL-12 or IL-10 were measured by ELISA test. 1-way ANOVA, Dunnett's corrected, ${ }^{* * *} \mathrm{p}<0,001$ vs untreated. C. Cells treated as in A were lysed and the expression of M-CSFR, COX-2, CD206, NOS2 and actin was evaluated by immunoblots. 
in driving tumour vascularization [8]. We therefore investigated whether the contact with ZA-treated M2/ M2-like-polarized macrophages, could affect the angiogenic features of endothelial cells. We treated HUVECs or EPCs with CM from the differently treated macrophages and assayed their ability to form tube-like structures in vitro. We showed that upon ZAtreatment there is a significant reduction of capillary-like structures assembly, suggesting that the drug, through the impairment of M2 polarization, may indirectly affect de novo intratumoral angiogenesis (Figure 3 and Supplementary Figure S3).

\section{$\mathrm{ZA}$ reverts $\mathrm{CAF}$-activated phenotype and their pro-invasive features}

To investigate the potential effects of ZA on other cellular components of tumour microenvironment, we focused our interest on stromal fibroblasts, since the achievement of aggressive features of cancer cells is strongly dependent on their contact with surrounding CAFs [2, 3]. Normal fibroblasts (HPFs) and CAFs were treated with increasing concentrations of ZA for 5 days. The evaluation of alpha smooth muscle actin $(\alpha-S M A)$ expression, a reported marker of fibroblasts
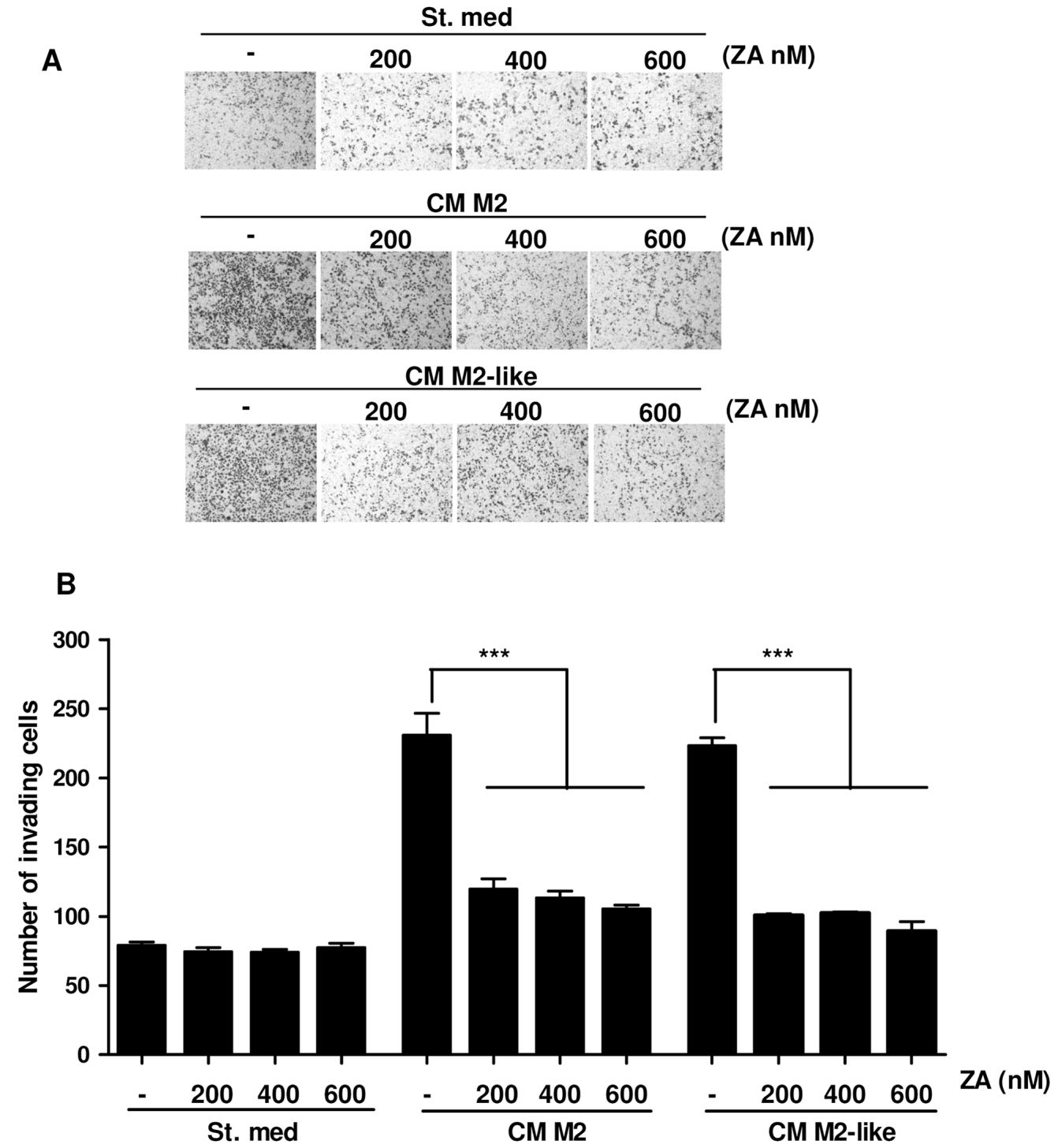

Figure 2: M2 and M2-like macrophages-dependent increase of PC3 cells invasiveness is impaired by ZA treatment. A. Monocytes were differentiated for 7 days with M-CSF and then polarized into M2 macrophages by stimulating with IL-4 for $24 \mathrm{~h}$. Alternatively, monocytes were stimulated with CM from PC3 for 7 days to obtain M2-like macrophages. ZA was administrated during differentiation at different concentrations and then macrophages were serum-starved for $48 \mathrm{~h}$ to obtain the corresponding CM. PC3 cells were incubated for $24 \mathrm{~h}$ with CM from the above differentiated macrophages (treated or not with ZA), or serum starved as a control, and then allowed to invade toward medium containing 10\% serum as chemoattractant for additional 24 h. (St. Med. Starvation Medium) B. Invading cells were counted and the mean of six randomly chosen fields was plotted in the bar graph. 1-way ANOVA, Dunnett's corrected, $* * * \mathrm{p}<0,001$ vs untreated. 
activation (Figure 4A) and the quantification of collagen contractility, an established feature of activated fibroblasts (Figure 4B), reveal that ZA administration completely reverts the activated phenotype of CAFs. To confirm the ZA-dependent conversion of CAFs towards a non-activated state, we explored whether ZA-treated CAFs were still able to promote cancer cell motility.
PC3 cells were conditioned with CM obtained from HPF and CAF treated as above, and tested for their migratory abilities (Supplementary Figure S1B). Crucially, ZAtreatment abolishes CAF ability to enhance PC3 cell invasiveness (Figure 4B-4C). Comparable results were obtained with DU145, an additional PCa cell line (Supplementary Figure S4).

A

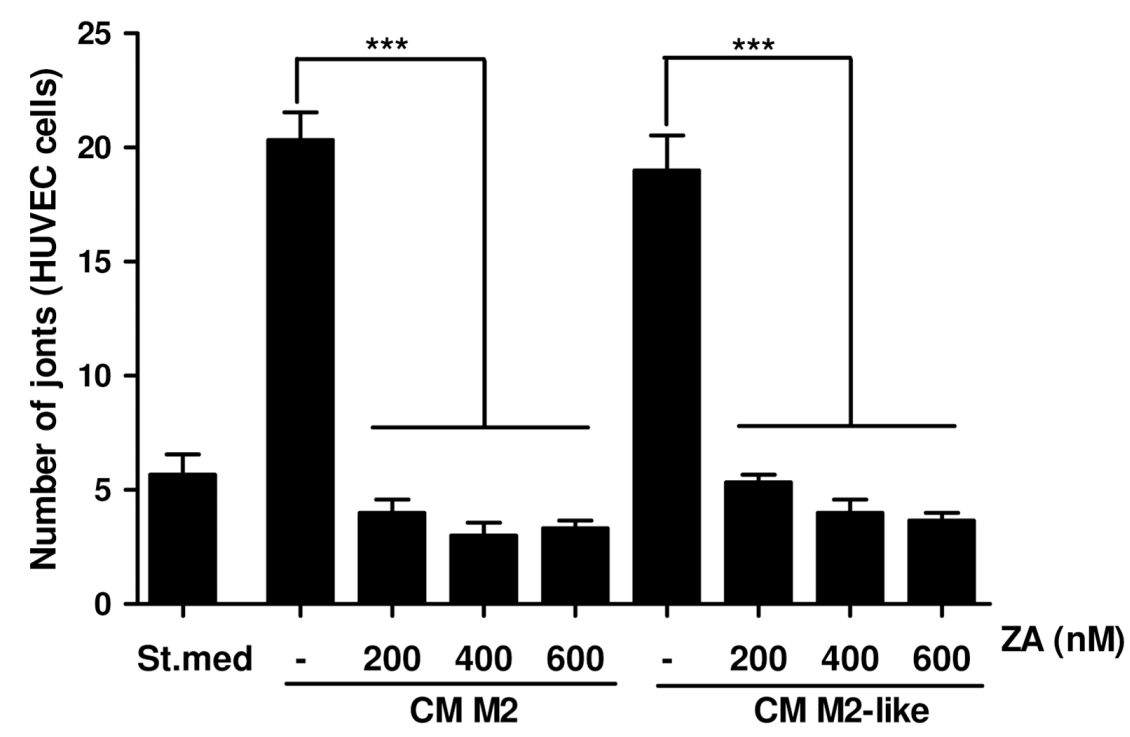

B

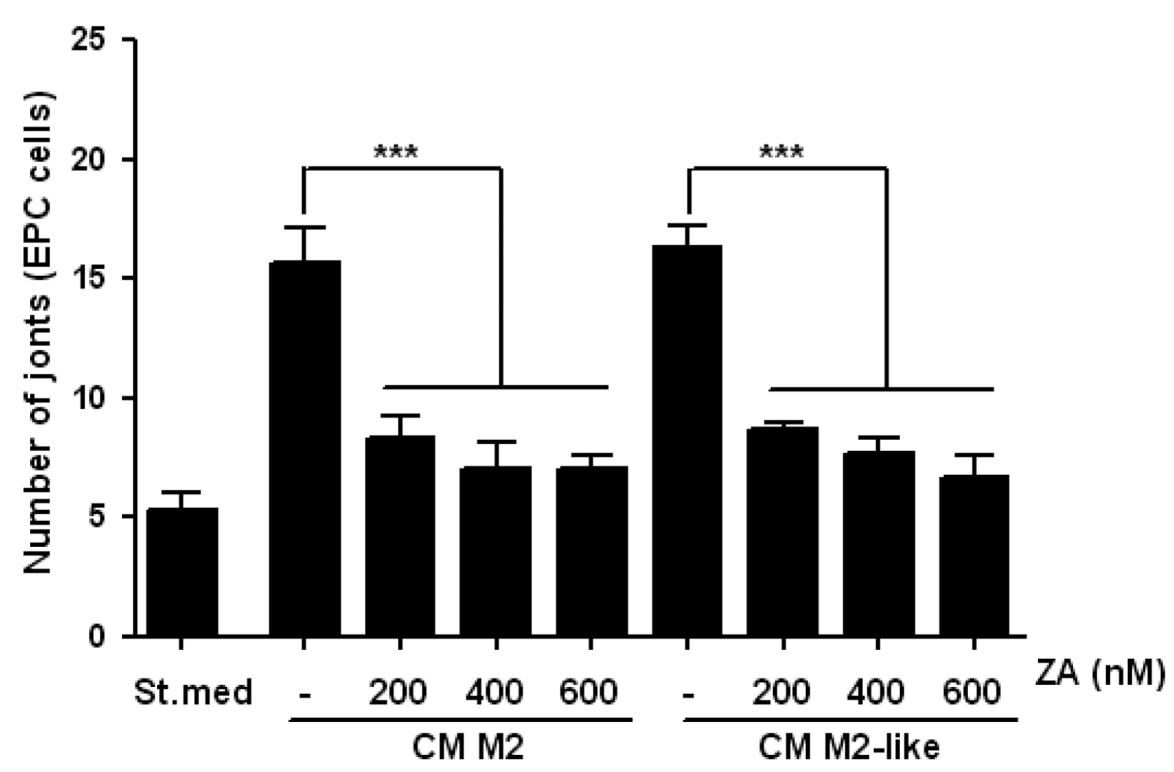

Figure 3: Treatment with $\mathrm{ZA}$ inhibits the M2/M2-like macrophages-induced pro-angiogenic effect. M2 and M2-like macrophages were obtained by treating monocytes for 7 days with M-CSF (then treated with IL-4 for additional $24 \mathrm{~h}$ ) or with CM from PC3, respectively. M2 and M2-like macrophages were treated with different concentrations of ZA during differentiation and then were serum-starved for $48 \mathrm{~h}$ to obtain the corresponding CM. HUVEC A. and EPC B. cells were treated with CM from the above differentiated macrophages (treated or not with ZA) and in vitro angiogenesis was evaluated by capillary morphogenesis assay. The number of joints was quantified and plotted in the bar graph. 1-way ANOVA, Dunnett's corrected, ***p<0,001 vs untreated. 
These data reveals that ZA impairs CAF activation and therefore their supportive role in PCa malignancy.

\section{ZA hinders fibroblast activation through the inhibition of RhoA-GTPase activity}

It has been reported that ZA inhibits the active site of the enzyme farnesyl pyrophosphate synthase in the mevalonate pathway, resulting in reduced levels of isoprenoids, including farnesyl pyrophosphate (FPP) and geranyl-geranyl pyrophosphate (GGPP), required for the prenylation of GTPase signalling proteins (i.e. Rac, Rho, Cdc42). This lipid modification is essential for the small GTPases family to achieve their active state and to ensure cytoskeletal rearrangements and dynamic cell shape remodelling [19]. To gain insight into the possible

A
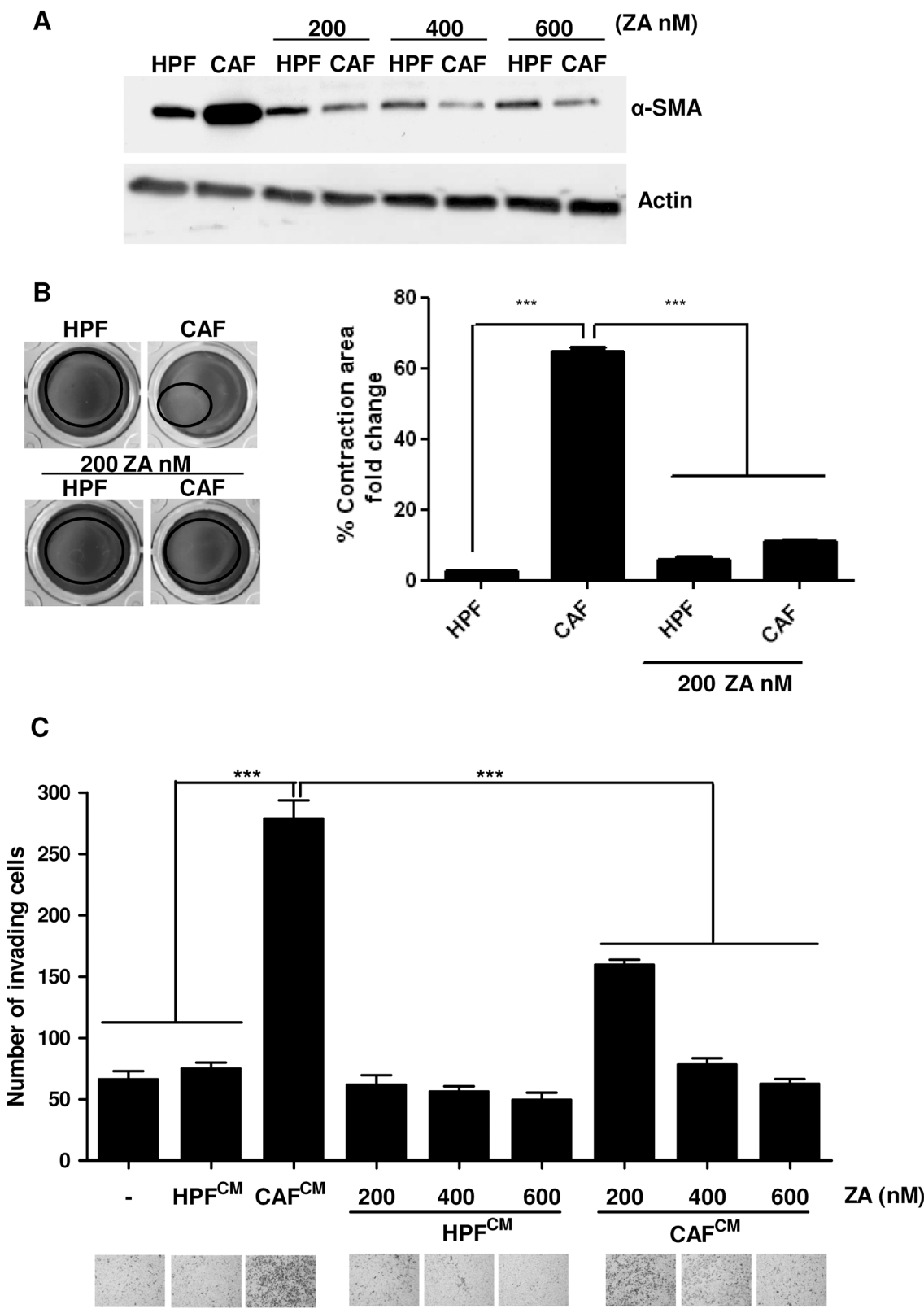

Figure 4: ZA administration reverts CAF activation and impairs their pro-invasive effects on cancer cells A. Subconfluent HPFs and CAFs were serum starved and treated for 5 days with different concentration of ZA. $\alpha$-SMA was analyzed by immunoblot as a marker for fibroblast activation. Actin was used as loading control. B. HPFs and CAFs were treated in serum-free medium with ZA 200nM for 5 days and after were placed in DMEM supplemented with collagen. Serum-starved HPFs and CAFs were used as control cells. Contraction of the collagen discs is expressed as relative collagen area from ST group. 1-way ANOVA, Bonferroni's corrected***p $<0,001$ vs CAF. C. PC3 cells were incubated for $24 \mathrm{~h}$ with CM from HPFs and CAFs treated as in A and then allowed to invade for additional 24 $\mathrm{h}$ toward medium containing $10 \%$ serum as chemoattractant. Invading cells were counted and the mean of six randomly chosen fields was plotted in the bar graph. 1-way ANOVA, Dunnett's corrected, ***p $<0,001$ vs CM CAF. 
molecular mechanism responsible for ZA-mediated reversion of CAFs activated state, we focused on the Rho family GTPases and we examined whether ZA was able to affect RhoA activity by reducing its geranylgeranylation. To evaluate RhoA activity, CAFs were treated in the presence or absence of $200 \mathrm{nM} Z \mathrm{ZA}$, the lower dose of the drug that is effective in the reversion of CAFs activated phenotype, and then a pull-down assay of active GTP-bound RhoA was performed. Our results indicate that $\mathrm{ZA}$ treatment reduces RhoA activity, while the exposure to GGPP or to the Rho activator calpeptin, concomitantly to ZA administration, restores the GTP-bound active state (Figure 5A). In addition, analysis of $\alpha$-SMA expression in our experimental setting

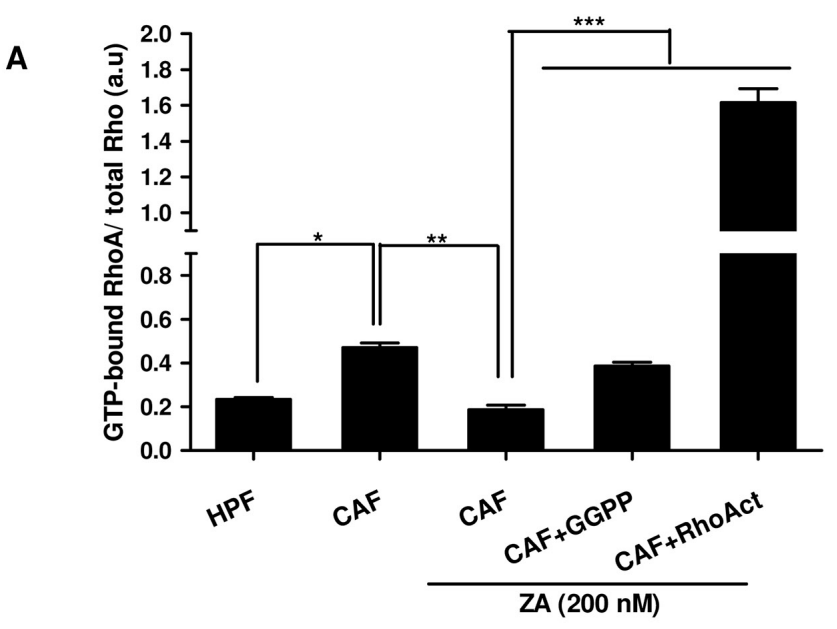

B
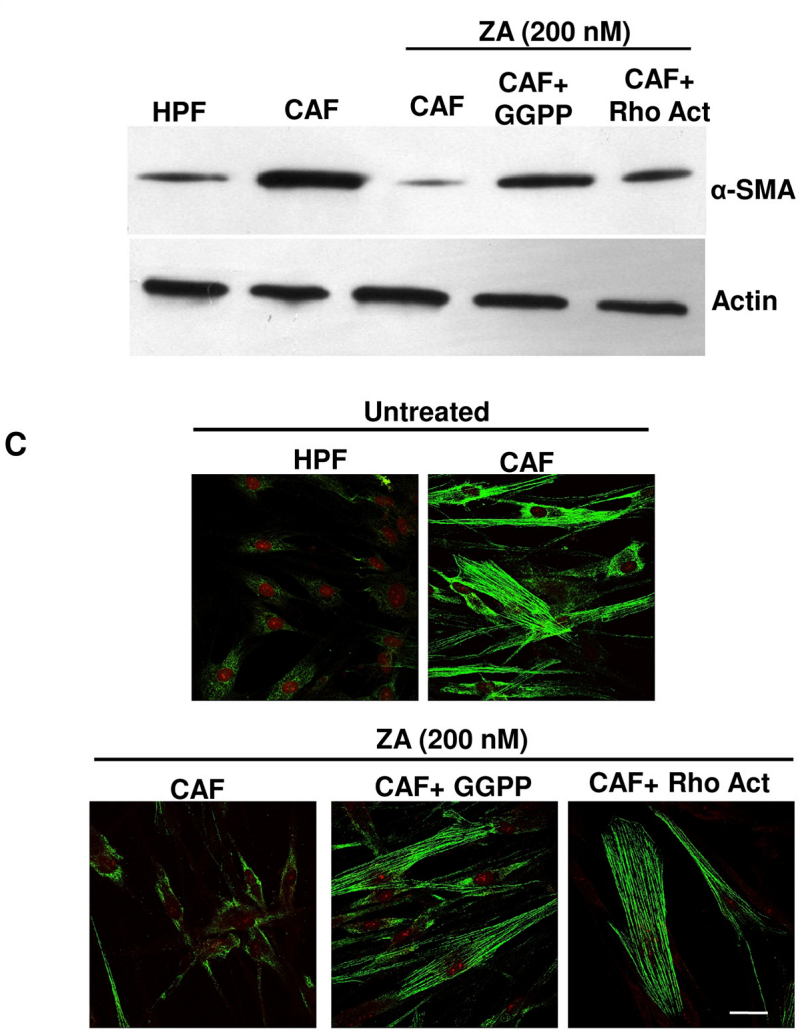

Figure 5: ZA inhibits RhoA prenylation/activation, thereby abrogating $\alpha$-SMA organization and CAF activation. A. CAFs were treated in serum-free medium with ZA 200nM for 5 days alone or in combination with geranylgeranyl pyrophosphate 20 $\mu \mathrm{M}$ (ZA+GGPP) or Rho Activator (ZA+RhoAct). Serum-starved HPFs and CAFs were used as control cells. ActiveGTP-bound RhoA was quantified by Rhotekin pull-down assay and normalized by total RhoA immunoblot upon densitometric analysis. 1-way ANOVA, Bonferroni's corrected, ${ }^{*} \mathrm{p}<0,05$ vs HPF, ${ }^{*}{ }^{*} \mathrm{p}<0,01$ vs CAF, ${ }^{* * *} \mathrm{p}<0,001$ vs CAF+ZA. B-C. Fibroblasts were treated as in A. $\alpha$-SMA was quantified by both immunoblot (B) and confocal microscopy. Bar, $50 \mu \mathrm{m}(\mathrm{C})$. 
underlines that RhoA signalling is critical for fibroblast activation. Indeed, ZA-mediated prevention of RhoA activation strongly reduces $\alpha$-SMA expression, while the addition of GGPP or Rho activator avoids $\alpha$-SMA downregulation, thus overcoming ZA-mediated mevalonate pathway inhibition (Figure 5B).

To corroborate these data, we also analysed $\alpha$-SMA stress fibers organization by confocal microscopy. While ZA-treated cells showed a faint signal with almost no stress fibers, GGPP or Rho activator administration recovered pronounced $\alpha$-SMA fibers formation (Figure $5 \mathrm{C}$ ), suggesting the role of ZA in impairing RhoA-mediated $\alpha$-SMA organization and stabilization of CAF phenotype.

\section{ZA abrogates macrophage-dependent fibroblast activation}

We recently demonstrated that $\mathrm{M} 2$ macrophages are able to activate healthy HPFs into CAFs, endowing them with supportive roles for PCa cells [8]. To better clarify the possible role of ZA in the complex interdependency among the different components of tumour microenvironment, we analysed the effects of ZA-treated M2-polarized macrophages on fibroblast activation. HPFs were incubated with $\mathrm{CM}$ from M2 or M2-like-macrophages, with or without different concentration of ZA. Fibroblast activation was then analysed by the evaluation of $\alpha$-SMA expression (Figure 6A), stress fibers organization (Figure $6 \mathrm{~B})$ and by assessing their pro-invasive effect on $\mathrm{PCa}$ cells (Figure 6C). As expected, while M2 and M2-like macrophages are active in eliciting fibroblast activation and enhancement of PC3 invasiveness, the exposure to ZA strongly prevents these effects. Therefore, ZA is not only capable to revert the activated state of CAFs, already engaged with pro-aggressive roles, but it is also effective in preventing the M2 macrophages-mediated activation of normal fibroblast, thereby strengthening the broad inhibitory function of this drug on tumour microenvironment.

\section{ZA prevents CAF-induced enhancement of PCa cell tumorigenicity and lung colonization in vivo}

As previously reported, CAF conditioning improves tumour growth in xenograft models and promotes lung colonization and metastases formation [2]. According to our in vitro results, we aimed to assess whether ZA could be able to neutralize the effects induced by CAFs on tumour growth and metastatic spread in vivo. As previously reported, we used PC3 cells in limiting conditions in order to ensure their in vivo growth and metastatic spread only when co-injected with CAFs [2, 3]. In particular, a mixture of CAFs with PC3 cells (2:1 ratio) was subcutaneously injected into the lateral flanks of SCID bg/bg mice. Mice received repeated courses of intraperitoneal injection of ZA $(100 \mu \mathrm{g} / \mathrm{kg})$ once a week. We observed that subcutaneous tumours of CAFs/ PC3 injected mice showed higher rate of tumour growth compared to ZA-treated tumours (Figure 7A). In addition, histological examination of lungs of tumour-bearing mice revealed the presence of spontaneous micrometastases in all the mice injected with the mixture CAFs/PC3. Conversely, ZA-treated animals exhibited a significant reduction in the number of metastatic areas (Figure $7 \mathrm{~B}$ ).

Comparable results were obtained with DU145 cells (Supplementary Figure S5). These data suggest that the clinical efficacy of ZA could be ascribed to its ability to rebuild a neutral microenvironment which counteracts tumour growth and metastatic spread.

\section{DISCUSSION}

ZA is a bisphosphonate now approved for use in the treatment of cancer-associated bone disease, preventing osteoclast-mediated bone breakdown. Indeed, ZA has been shown to effectively reduce the risk of skeletal-related events in patients with metastatic PCa [20], multiple myeloma [21] and breast cancer [20, 22, 23]. ZA has also recently been tested as an additive therapeutic for earlystage breast cancer [24-26].

In addition to its standard application in the adjuvant treatment of symptomatic bone metastases, several evidence about a direct anti-tumour effect of ZA are emerging [27]. It has been reported that bisphosphonates possess calcium-chelating properties, responsible for the bone-binding properties of these compounds. Since the dysregulation of $\mathrm{Ca}^{2+}$ homeostasis has been suggested as an important event in driving the enhancement of malignant traits, such as cell proliferation, migration and invasion [28], as well as tumor vascularization [29], the ZA-dependent regulation of calcium homeostasis may be responsible, at least in part, for its anti-tumor activity. Notably, ZA also attracts interest as a potent immunomodulator, which can be exploited in cancer immunotherapy. Recently, Coscia and colleagues reported that administration of clinically compatible doses of ZA is an effective way of targeting hyperplastic cells and TAMs by switching the local microenvironment from a highly permissive partner to a tumour-hostile counterpart in a murine breast cancer [16]. The anti-tumour effect of ZA also relies on the ability of the drug to impair the mesenchymal stem cellsinduced TAMs recruitment to tumour sites by decreasing the expression of monocyte chemotactic protein-1 (MCP-1), thus resulting in tumour growth inhibition [30]. In addition, ZA has been reported to cooperate with different chemotherapeutic agents (e.g. docetaxel, imatinib, cisplatin) in impairing cancer cells growth in vitro or in vivo [31-34].

Although the effects of ZA are clinically well known, the molecular mechanism responsible for its effect on cancer cells are not yet well elucidated. 
Here, we underscore a role of the compound on tumour microenvironment focusing on the profound impact exerted by ZA on M2 macrophages and prostate CAFs. According to our evidence, we suggest that the anti-tumour effect of ZA relies on the direct targeting of stromal and immune cells and on the prevention of their pro-tumoral skills.

Resident and recruited cells which infiltrate prostate tumours engage with cancer cells a complex network of relationships that evolve alongside

A

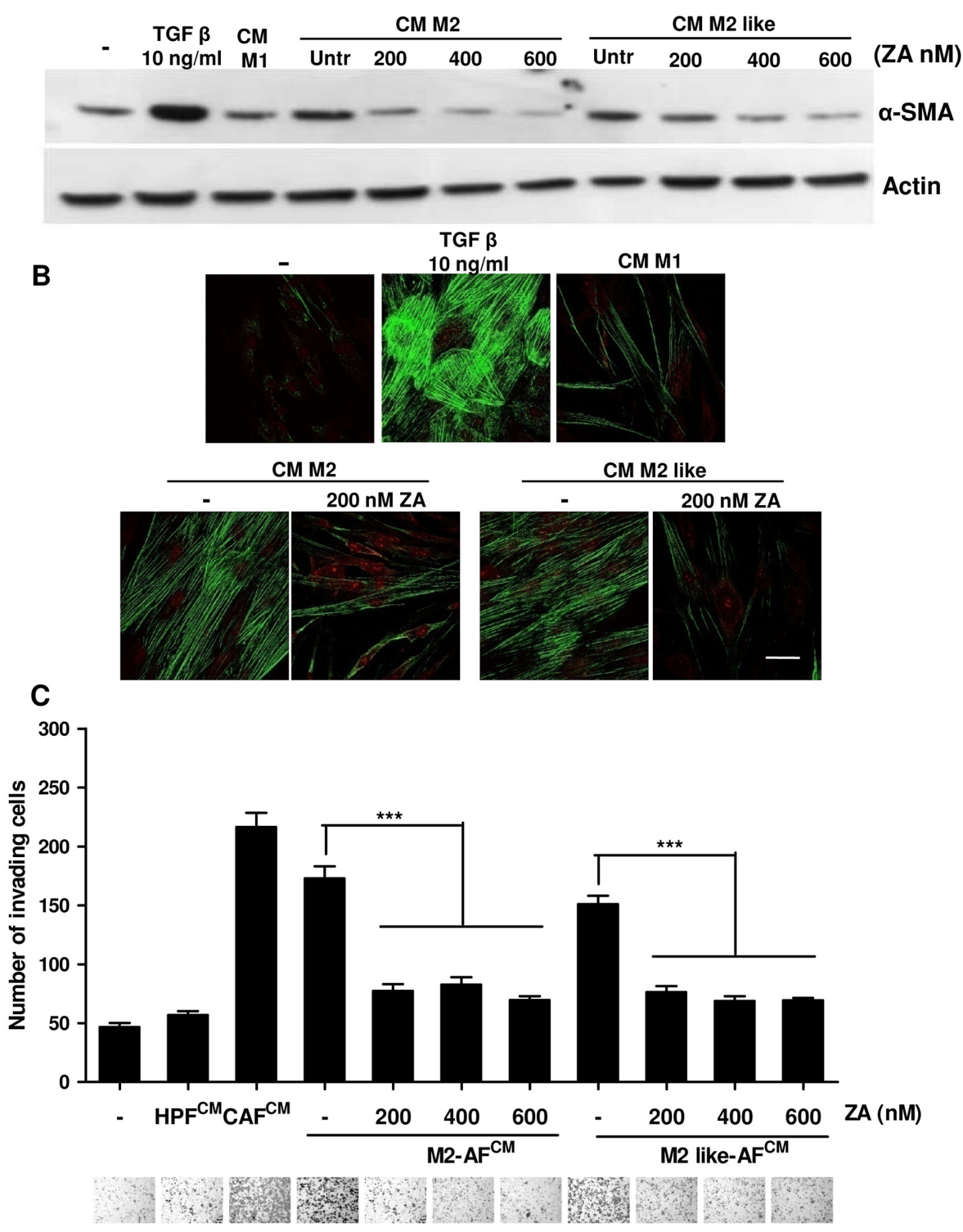

Figure 6: ZA counteracts macrophage-dependent fibroblasts activation. A. M2 and M2-like macrophages were obtained by treating monocytes for 7 days with M-CSF (then treated with IL-4 for additional 24h) or with CM from PC3, respectively. M2 and M2-like macrophages were treated with different concentrations of ZA during differentiation and then were serum-starved for $48 \mathrm{~h}$ to obtain the corresponding CM. Subconfluent HPFs were treated for $24 \mathrm{~h}$ with $\mathrm{CM}$ from the above differentiated macrophages (treated or not with ZA) or with $10 \mathrm{ng} / \mathrm{ml} \mathrm{TGF-} \beta 1$ as a positive control, and serum starved for additional $24 \mathrm{~h}$. Fibroblasts were lysed and immunoblots for $\alpha$-SMA and actin were performed. B. M2 and M2-like macrophages were obtained as in A and were treated with ZA 200nM during differentiation and then were serum-starved for $48 \mathrm{~h}$ to obtain the corresponding CM. Subconfluent HPFs were treated for $24 \mathrm{~h}$ with CM from the above differentiated macrophages (treated or not with ZA) or with $10 \mathrm{ng} / \mathrm{ml}$ TGF- $\beta 1$ as a positive control, $\alpha$-SMA was quantified by confocal microscopy. Bar, $50 \mu \mathrm{m}$. C. PC3 cells were incubated for $24 \mathrm{~h}$ with CM from HPFs treated as in A (CM M2 AFs or CM M2-like AFs; AFs: Activated fibroblasts) and then allowed to invade for additional $24 \mathrm{~h}$ toward medium containing $10 \%$ serum as chemoattractant. Invading cells were counted and the mean of six randomly chosen fields was plotted in the bar graph. Representative photographs for each sample are shown under the corresponding bar. 1-way ANOVA, Dunnett's corrected, ***p<0,001 vs untreated. 
malignant progression [35]. Among several cell types affecting PCa behaviour and malignancy, CAFs and macrophages exert important and overlapping roles $[35,36]$. Recently, we demonstrated that CAFs and TAMs cooperate to promote PCa malignancy. Indeed, CAFs promote monocyte trans-differentiation into the M2 macrophage phenotype. In turn, this pro-tumoral subset of macrophages fosters stromal reactivity, by inducing fibroblast activation. This positive loop established between immune and stromal components is also supported by tumour cells, which participate to this cross-talk through secretion of MCP-1 and IL6 , thereby facilitating both monocyte recruitment and again differentiation into M2 macrophages [8].

A

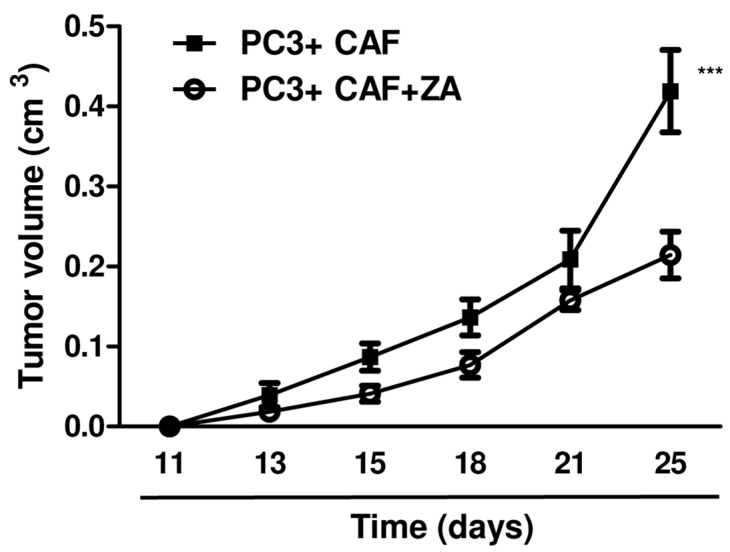

B

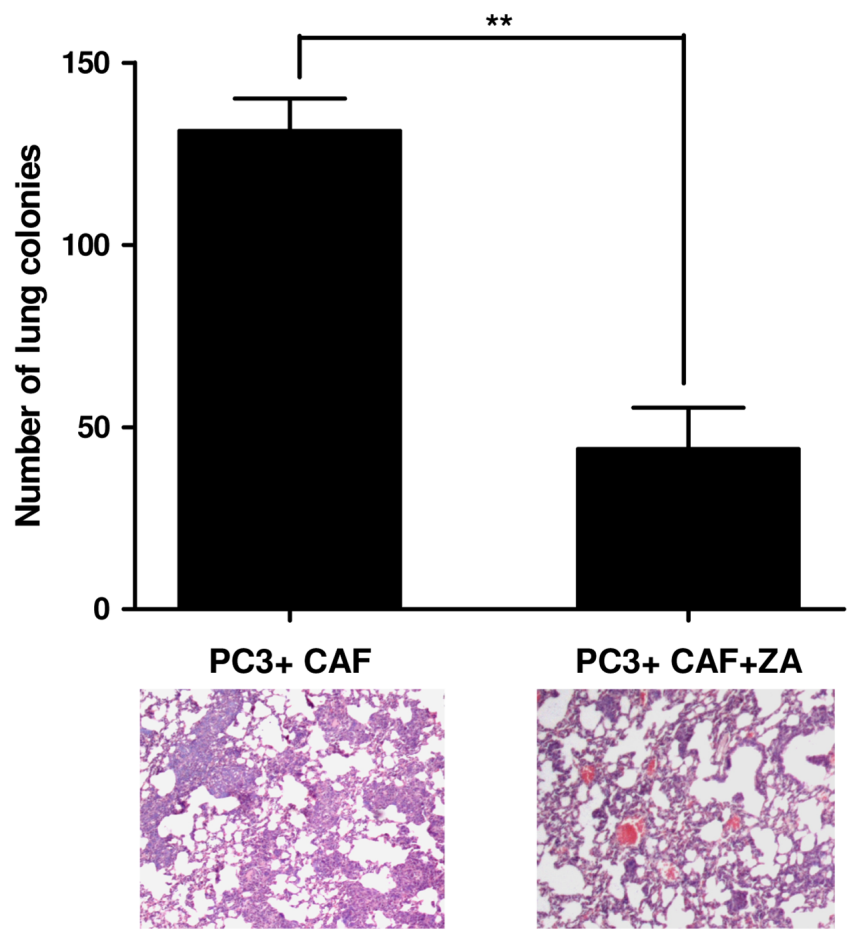

Figure 7: ZA in vivo administration prevents tumor growth and lung metastatic dissemination. A. A mixture of $1 \times 10^{6}$ PC3 cells and $0.5 \times 10^{6}$ CAFs were subcutaneously injected in both the lateral flanks of SCID bg/bg mice. Mice were treated once a week with intraperitoneal injection of PBS (control mice) or $100 \mu \mathrm{g} / \mathrm{kg}$ of ZA, for 6 weeks ( $\mathrm{n}=5$ per group), starting the treatment 24 $\mathrm{h}$ after cell injection. The volume of the primary tumour for control or ZA-treated mice was reported at different time points. 2-way ANOVA, Bonferroni's corrected ${ }^{* * *} \mathrm{p}<0,001$ vs untreated B. Mice were sacrificed after 6 weeks. Lungs were inspected and the number of micrometastases were counted and plotted. Representative paraffin-embedded lung tissue sections, stained with hematoxylin/eosin, are shown. Student t-test, $* *$ p $<0,0036$ vs PC3+CAF. 
Here, we demonstrate that M2 polarization is reversed by ZA treatment, which aborted IL-10 production, while it is ineffective on the recovery of the M1-polarized macrophages. In contrast, recent evidence suggest that therapeutic concentrations of $\mathrm{ZA}$ affect all types of macrophages, reinforcing the idea that the anti-metastatic effects of ZA are predominantly caused by modulating tumour microenvironment [37]. While M1-polarized macrophages are likely to play an antitumoral response, M2-polarized macrophages have been associated with a chronic inflammatory response that characterizes several highly malignant tumours $[12,38]$. The ZA-dependent neutralization of M2 pro-tumoral activity brings to three main consequences: i) loss of M2 macrophages mediated enhancement of PCa cell motility; ii) reduction of stromal reactivity, due to the failure of $\mathrm{M} 2$ macrophages to trigger fibroblast activation; iii) inhibition of endothelial cells organization into capillary-like structures, to which cooperate both TAMs and CAFs. The overall effect is to counteract cancer cells motile spur as well as tumour vascularization, ultimately avoiding cancer cell escape from the primary site and preventing metastatic dissemination.

Besides the inhibitory effect of ZA on fibroblast activation via M2 macrophages neutralization, we also highlight a direct function of the drug in reverting the already established CAF activated phenotype. Indeed, ZA administration impairs $\alpha$-SMA expression as well as its organization in stress fibers, required to enhance cell contractility. These ZA-induced effects are mainly due to the interference of the drug with the mevalonate-dependent isoprenoid metabolism [39]. Indeed, the failure in the posttranslational addition of GGPP to RhoA impairs the correct localization and function of the small GTPase, thereby affecting actin cytoskeleton remodelling, stress fibers formation and ultimately cell contractility. In keeping, previous studies showed that inhibition of the mevalonate pathway by statins prevents TGF- $\beta$-induced myofibroblast transdifferentiation in human Tenon fibroblasts interfering with Rho signalling [40]. Since re-exposure to GGPP rescued ZA-dependent inhibition of a-SMA, both in terms of expression and subcellular localization, we propose RhoA geranylgeranylation as responsible for the positive effect of the mevalonate pathway on the maintenance of CAFs active state.

Alterations within the microenvironment in which the tumor develops are now recognized to be crucial during key steps of tumor progression, making tumor microenvironment components attractive candidates for therapeutic modulation. Given the tumour-supporting function elicited by CAFs and the self-reinforcing loop established between cancer and stromal cells to promote cancer cell aggressiveness, a strategy aimed at suppressing CAF activation could have interesting therapeutic implications. It has been recently reported that bisphosphonates reduce the rate of breast cancer recurrence in bone, according to a meta-analysis of randomized trials of bisphosphonates as adjuvant therapy for women with early-stage breast cancer. In particular, addition of ZA to adjuvant endocrine therapy reduced disease recurrence in bone and non-bone sites [26]. According to our evidence, a possible explanation of the efficacy of ZA could be ascribed to the effect of this bisphosphonate on CAF deactivation, through the inhibition of RhoA geranyl-geranylation. We speculate that ZA-induced stromal neutralization could interfere with the supporting role of CAFs in triggering cancer cell escaping from primary site. It is conceivable that the synergistic effect observed by combining ZA administration to conventional adjuvant endocrine therapy could rely on the double inhibitory effect on cancer cell proliferation (exerted by endocrine therapy) and metastatic dissemination (induced by ZA treatment). CAF neutralization induced by ZA may also prevent the onset of chemoresistance, thereby favoring response to conventional therapy.

Other indications strengthen the idea that interfering with CAF active state and promoting stromal normalization may be a potential antitumor approach. In particular, Mitra et al. previously reported that mimicking deregulation of miR-31, miR-214 and miR-155 resulted in the reversion of ovarian CAFs into normal fibroblasts, due to the down-regulation of several chemokines, which are known to be important for CAF function [41]. More recently, it has been proposed that prolonged exposure to hypoxia, through a prolyl hydroxylase domain protein 2-dependent stabilization of hypoxia-inducible factor-1a, counteracts a-SMA expression, ultimately leading to CAF deactivation [42].

Our results globally highlight that ZA exerts a specific targeting of stromal inflammatory cells, thus hindering their direct pro-tumoral effect and their mutual activation loop. In particular, we observe that ZA neutralizes pro-invasive and pro-angiogenic activity of M2 macrophages and fascinatingly leads to CAF deactivation, thereby strongly reducing cancer malignancy both in vitro and in vivo. These findings support ZA efficacy outside bone environment and provide an additional evidence of $\mathrm{ZA}$ as a potential approach to prevent cancer progression through abrogating the metastasis-promoting effects of tumour microenvironment.

\section{MATERIALS AND METHODS}

\section{Materials}

Unless specified, all reagents were obtained from Sigma. Antibodies against COX-2, MCSF-R and $\beta$-Actin was from Santa Cruz Biotechnology. TGF- $\beta 1$, MCSF and IL-4 were from Peprotech. Rho Activator CN01 (calpeptin) was from Cytoskeleton, Inc. PVDF was from Millipore and Matrigel and GST-Rhotekin were from BD 
Biosciences. The ELISA kit for the quantification of IL10 and IL-12 was from Invitrogen. ZOMETA (Zoledronic acid) was from Novartis.

\section{Cell cultures}

Human prostate carcinoma cells (PC3 and DU145) and human umbilical vein endothelial cells (HUVEC) were obtained and authenticated by PCR/ short tandem repeat (STR) analysis from the European Collection of Cell Cultures (ECACC). PC3 cells were maintained at $37^{\circ} \mathrm{C}$ and $5 \% \mathrm{CO} 2$ in DMEM supplemented with $10 \%$ fetal bovine serum, while HUVEC were cultured on gelatin 1\% coated dishes in EGM-2 medium (Lonza). Endothelial progenitor cells (EPCs) have been isolated from human umbilical cord blood as previously described [43, 44]. Human prostate fibroblasts (HPFs and CAFs) were isolated from surgical explantation after patient informed consent in accord with the Ethics Committee of the Azienda Ospedaliera Universitaria Careggi. Briefly, HPFs and CAFs were extracted from healthy and intratumoral regions of prostate of $\mathrm{PCa}$-bearing patients with mean Gleason Score $9(4+5)$ and clinical stage T3. Tissue samples were aseptically obtained from patients undergoing radical prostatectomy with final pathological examination confirmation of pT3aN0 disease. Tissues were digested overnight in $1 \mathrm{mg} /$ $\mathrm{ml}$ collagenase I and cells were plated in DMEM containing $10 \%$ fetal bovine serum [2].

\section{Monocyte isolation and macrophages differentiation}

Human monocytes were obtained from normal donor buffy coat by gradient centrifugation using Ficoll (Histopaque-1077). Non-adherent cells were removed and purified monocytes were incubated for 7 days in RPMI 1640 supplemented with 10\% FBS and $50 \mathrm{ng} / \mathrm{ml}$ macrophage-colony stimulating factor (M-CSF) to obtain macrophages. M1 macrophages were polarized by stimulating overnight with LPS (100 ng/ $\mathrm{ml}$ ) (Peprotech) and IFN $\gamma(100 \mathrm{ng} / \mathrm{ml})$ (Peprotech). M2 macrophages were polarized by stimulating overnight with interleukin-4 (IL-4) $(20 \mathrm{ng} / \mathrm{ml})$ (Peprotech). M2like macrophages were obtain by culturing monocytes for 7 days in RPMI $164010 \%$ FBS with 50\% of conditioned medium from PCa.

\section{Preparation of conditioned media}

Conditioned media (CM) were obtained from untreated HPFs, CAFs, macrophages or PCa. Cells were grown to sub-confluence, then serum starved and incubated for $48 \mathrm{~h}$ before collection of the CM. CM were harvested, clarified by centrifugation, and used freshly.

\section{Cell lysis and western blot analysis}

Fibroblasts, PCa cells or macrophages derived from our experimental conditions were lysed for 20 min on ice in $500 \mu \mathrm{l}$ of RIPA lysis buffer $(50 \mathrm{mM}$ Tris- $\mathrm{HCl}, \mathrm{pH} 7.5,150 \mathrm{mM} \mathrm{NaCl}, 1 \%$ Triton X-100, $2 \mathrm{mM}$ EGTA, $1 \mathrm{mM}$ sodium orthovanadate, $1 \mathrm{mM}$ phenylmethanesulphonyl-fluoride, $10 \mathrm{mg} / \mathrm{ml}$ aprotinin, $10 \mathrm{mg} / \mathrm{ml}$ leupeptin). $20 \mu \mathrm{g}$ of total proteins were loaded on SDS-PAGE, separated and transferred onto nitrocellulose. The immunoblots were incubated in 3\% bovine serum albumin, $10 \mathrm{mmol} / \mathrm{L}$ Tris- $\mathrm{HCl}(\mathrm{pH} 7.5)$, $1 \mathrm{mmol} / \mathrm{L}$ EDTA, and $0.1 \%$ Tween 20 for $1 \mathrm{~h}$ at room temperature, probed first with specific antibodies and then with appropriate secondary antibodies.

\section{In vitro boyden invasion assay}

PCa cell invasion was assayed with the Transwell system of Costar equipped with $8 \mu \mathrm{m}$-pore-size polyvinylpyrrolidone- free polycarbonate filters. Migration or invasiveness assays are distinguished by the absence (migration assay) or the presence (invasiveness assay) of a 3D barrier of Matrigel. $50 \mu \mathrm{g} / \mathrm{cm}^{2}$ of reconstituted Matrigel (BD Biosciences) was added to the top chamber, allowed to solidify for $1 \mathrm{~h}$ at $37^{\circ} \mathrm{C}$, and air dried for 16 h. The Matrigel barrier was rehydratated with $100 \mu \mathrm{l}$ of Dulbecco's modified Eagle's medium for $2 \mathrm{~h}$ at $37^{\circ} \mathrm{C}$ prior to use. Cells were loaded into the upper compartment ( 5 $\mathrm{x} 10^{4}$ cells in $200 \mu \mathrm{l}$ ) in serum-deprived growth medium. The Matrigel invasion chambers were placed into 24well culture dishes containing $500 \mu \mathrm{l}$ of the different $\mathrm{CM}$ from monocytes as a chemoattractant. After $24 \mathrm{~h}$ of incubation at $37^{\circ} \mathrm{C}$, non-invading cells and the Matrigel layer were mechanically removed using cotton swabs, and the microporous membrane containing the invaded cells was fixed in 96\% methanol and stained with DiffQuick staining solutions. Chemotaxis was evaluated by counting the cells that migrated to the lower surfaces of the polycarbonate filters.

\section{Flow cytometer analysis of apoptotic cell death}

Cells undergoing apoptosis were assayed by the Annexin-V FLUOS Staining Kit (Roche Applied Sciences), according to the manufacturer's instructions. Briefly, $5 \times 10^{5}$ PC3 cells were washed with ice-cold PBS and labeled for 10 min with both FITC-labelled Annexin V and Propidium iodide (PI) in $100 \mathrm{ml}$ of binding buffer (HEPES-buffered saline solution with $2.5 \mathrm{mM} \mathrm{CaCl}$ ). Flow cytometry was performed using a FACSscan (BD Biosciences, San Jose, CA, USA). The analyzer threshold was adjusted on the flow cytometer channel to exclude most of the subcellular debris to reduce the background noise. The totality of Annexin $\mathrm{V}+/ \mathrm{PI}$ - (early apoptotic) and Annexin V+/PI+ cells (late apoptotic) were considered apoptotic. 


\section{ELISA}

Cytokine production in macrophages supernatants were measured by commercially available ELISA Kits (IL-10 and IL-12) according to the manufacturer's instructions (Invitrogen KHC0104/ KHC0104).

\section{Tube-like formation assay}

All the experiments were performed using growth factor-reduced Matrigel at a concentration of $1 \mathrm{mg} / \mathrm{mL}$. $50 \mu \mathrm{L}$ of Matrigel were added to each well of a 96-well plate and then placed in a humidified incubator at $37^{\circ} \mathrm{C}$ for $30 \mathrm{~min}$. HUVECs and EPCs $\left(2 \times 10^{4}\right.$ cells/ well $)$ were added to the Matrigel-coated plates in a final volume of $200 \mu \mathrm{L}$. The effects on the morphogenesis of endothelial cells were recorded after $6 \mathrm{~h}$ with an inverted microscope equipped with CCD optics and a digital analysis system. Results were quantified by measuring the joint numbers in the field.

\section{Collagen gel contraction assay}

$1.5 \times 10^{5}$ fibroblasts were embedded in $500 \mu \mathrm{l}$ of DMEM solution containing $1 \mathrm{mg} / \mathrm{mL}$ collagen and cast into a well of 24-well plate (Corning, Costar). Such fibroblasts containing gel was allowed to polymerize at $37^{\circ} \mathrm{C}$ for $1 \mathrm{~h}$ before detaching the gel disc from the wall using a sterile tip. Subsequently, $1 \mathrm{ml}$ of serum-free DMEM with or without different concentration of ZA was added in the different wells. The floating gels were incubated at $37^{\circ} \mathrm{C}$ and $5 \% \mathrm{CO}_{2}$ for $24 \mathrm{~h}$ and the gel area were then quantified. To determine the degree of collagen gel contraction, pictures of the gels were taken and the estimated area of each gel (number of pixels) was analyzed with Adobe Photoshop ${ }^{\text {TM }}$.

\section{RhoA activity assay}

HPFs or differently treated CAFs were directly lysed in RIPA buffer, the lysates were clarified by centrifugation and RhoA-GTP were quantified. Briefly, lysates were incubated with $10 \mu \mathrm{g}$ Rhotekin-GST fusion protein (BD Biosciences) for $1 \mathrm{~h}$ at $4^{\circ} \mathrm{C}$. RhoA-GTP (on pull-down samples) and RhoA total content (on cell lysates) were then quantified by immunoblot.

\section{Confocal microscopy}

HPFs or differentially treated CAFs were fixed in p-formaldehyde (4\% v/v in PBS) for $20 \mathrm{~min}$. The cells were permeabilized in Triton X-100 $(0.5 \% \mathrm{v} / \mathrm{v}$ in PBS) for $5 \mathrm{~min}$, and then incubated in horse serum $(5 \% \mathrm{v} / \mathrm{v}$ PBS) for $30 \mathrm{~min}$, and with primary antibodies against $\alpha$-SMA (mouse monoclonal; 1:100; Sigma-Aldrich), overnight at $4^{\circ} \mathrm{C}$. After two washing steps with PBS, cells were incubated with anti-mouse AlexaFluor 488 (1:1000;
Molecular Probes) for $1 \mathrm{~h}$ at RT in the dark. The coverslips were mounted in Gel Mount ${ }^{\mathrm{TM}}$ Aqueous Mounting Medium (Sigma-Aldrich). A Leica SP5 Confocal microscope was used for data acquisition. Images were generated with Leica SP5 Confocal Software.

\section{Xenograft experiments}

In vivo experiments were performed in accordance with national guidelines and approved by the ethical committee of Animal Welfare Office of Italian Work Ministry and conform to the legal mandates and Italian guidelines for the care and maintenance of laboratory animals. 6- to 8-week-old male severe combined immunodeficient (SCID)-bg/bg mice (Charles River Laboratories International) were injected s.c. with $1 \times 10^{6} \mathrm{PC} 3$ cells plus $0.5 \times 10^{6} \mathrm{CAFs}$, both in the right and left lateral flanks. Mice received repeated courses of intra-peritoneal saline solution (control mice) or $100 \mu \mathrm{g} / \mathrm{kg}$ of ZA, once a week for 6 weeks. Animals (5 per group) were monitored daily. Tumour size was measured every 2 to 3 days by a caliper; tumor volumes were determined by the length (L) and the width (W): $\mathrm{V}=(\mathrm{LW} 2) / 2$. Mice were sacrificed after 6 weeks. Lungs were inspected for metastatic nodules by histological analyses.

\section{Histology}

Lungs were fixed in $4 \%$ ( $\mathrm{vol} / \mathrm{vol})$ phosphatebuffered formalin and paraffin embedded. Five longitudinal sections of each lung per mice ( $5 \mu \mathrm{m}$ thick) were mounted on positively charged slides. Tissue sections were deparaffinized, re-hydrated and stained with hematoxylin-eosin staining. Metastases were counted using NIS ELEMENTS_F-2.20 software combined with a microscope Nikon Eclipse 50i.

\section{Statistical analysis}

Data are presented as mean \pm SD from at least three independent experiments. Statistical analysis of the data was performed using GraphPad Prism and the relevant analyses are indicated in the Figure Legends.

\section{ACKNOWLEDGMENTS}

The work was supported by Associazione Italiana Ricerca sul Cancro (AIRC) and Istituto Toscano Tumori (ITT). We thank Azienda Ospedaliera Universitaria Careggi for providing ZOMETA for the in vitro and in vivo experiments.

\section{CONFLICTS OF INTEREST}

The authors declare no conflict of interest. 


\section{REFERENCES}

1. Cirri P, Chiarugi P. Cancer-associated-fibroblasts and tumour cells: a diabolic liaison driving cancer progression. Cancer Metastasis Rev. 2012; 31: 195-208.

2. Giannoni E, Bianchini F, Masieri L, Serni S, Torre E, Calorini L and Chiarugi P. Reciprocal activation of prostate cancer cells and cancer-associated fibroblasts stimulates epithelial-mesenchymal transition and cancer stemness. Cancer Res. 2010; 70: 6945-6956.

3. Giannoni E, Bianchini F, Calorini L, Chiarugi P. Cancer associated fibroblasts exploit reactive oxygen species through a proinflammatory signature leading to epithelial mesenchymal transition and stemness. Antioxid Redox Signal. 2011; 14: 2361-2371.

4. Wan L, Pantel K, Kang Y. Tumor metastasis: moving new biological insights into the clinic. Nat Med. 2013; 19: 1450-1464.

5. Giannoni E, Taddei ML, Parri M, Bianchini F, Santosuosso M, Grifantini R, Fibbi G, Mazzanti B, Calorini L and Chiarugi P. EphA2-mediated mesenchymal-amoeboid transition induced by endothelial progenitor cells enhances metastatic spread due to cancer-associated fibroblasts. J Mol Med (Berl). 2013; 91: 103-115.

6. Fiaschi T, Chiarugi P. Oxidative stress, tumor microenvironment, and metabolic reprogramming: a diabolic liaison. Int J Cell Biol. 2012; 2012: 762825.

7. Giannoni E, Taddei ML, Morandi A, Comito G, Calvani M, Bianchini F, Richichi B, Raugei G, Wong N, Tang D and Chiarugi P. Targeting stromal-induced pyruvate kinase M2 nuclear translocation impairs oxphos and prostate cancer metastatic spread. Oncotarget. 2015; 6: 24061-24074. doi: 10.18632/oncotarget.4448.

8. Comito G, Giannoni E, Segura CP, Barcellos-de-Souza P, Raspollini MR, Baroni G, Lanciotti M, Serni S and Chiarugi P. Cancer-associated fibroblasts and M2-polarized macrophages synergize during prostate carcinoma progression. Oncogene. 2014; 33: 2423-2431.

9. Qian BZ, Pollard JW. Macrophage diversity enhances tumor progression and metastasis. Cell. 2010; 141: 39-51.

10. Ruffell B, Affara NI, Coussens LM. Differential macrophage programming in the tumor microenvironment. Trends Immunol. 2012; 33: 119-126.

11. Hao NB, Lu MH, Fan YH, Cao YL, Zhang ZR, Yang SM. Macrophages in tumor microenvironments and the progression of tumors. Clin Dev Immunol. 2012; 2012: 948098.

12. Sica A, Mantovani A. Macrophage plasticity and polarization: in vivo veritas. J Clin Invest. 2012; 122: 787-795.

13. Valdespino V, Tsagozis P, Pisa P. Current perspectives in the treatment of advanced prostate cancer. Med Oncol. 2007; 24: 273-286.
14. Kuroda J, Kimura S, Segawa H, Kobayashi Y, Yoshikawa T, Urasaki Y, Ueda T, Enjo F, Tokuda H, Ottmann OG and Maekawa T. The third-generation bisphosphonate zoledronate synergistically augments the anti- $\mathrm{Ph}+$ leukemia activity of imatinib mesylate. Blood. 2003; 102: 2229-2235.

15. Yamada J, Tsuno NH, Kitayama J, Tsuchiya T, Yoneyama S, Asakage M, Okaji Y, Shuno Y, Nishikawa T, Tanaka J, Takahashi K and Nagawa H. Anti-angiogenic property of zoledronic acid by inhibition of endothelial progenitor cell differentiation. J Surg Res. 2009; 151: 115-120.

16. Coscia M, Quaglino E, Iezzi M, Curcio C, Pantaleoni F, Riganti C, Holen I, Monkkonen H, Boccadoro M, Forni G, Musiani P, Bosia A, Cavallo F et al. Zoledronic acid repolarizes tumour-associated macrophages and inhibits mammary carcinogenesis by targeting the mevalonate pathway. J Cell Mol Med. 2010; 14: 2803-2815.

17. Giraudo E, Inoue M, Hanahan D. An amino-bisphosphonate targets MMP-9-expressing macrophages and angiogenesis to impair cervical carcinogenesis. J Clin Invest. 2004; 114 : 623-633.

18. Mantovani A, Sica A. Macrophages, innate immunity and cancer: balance, tolerance, and diversity. Curr Opin Immunol. 2010; 22: 231-237.

19. Roelofs AJ, Thompson K, Gordon S, Rogers MJ. Molecular mechanisms of action of bisphosphonates: current status. Clin Cancer Res. 2006; 12: 6222s-6230s.

20. Lipton A, Small E, Saad F, Gleason D, Gordon D, Smith M, Rosen L, Kowalski MO, Reitsma D and Seaman J. The new bisphosphonate, Zometa (zoledronic acid), decreases skeletal complications in both osteolytic and osteoblastic lesions: a comparison to pamidronate. Cancer Invest. 2002; 20: 45-54.

21. Morgan GJ, Davies FE, Gregory WM, Cocks K, Bell SE, Szubert AJ, Navarro-Coy N, Drayson MT, Owen RG, Feyler S, Ashcroft AJ, Ross F, Byrne J, et al. Firstline treatment with zoledronic acid as compared with clodronic acid in multiple myeloma (MRC Myeloma IX): a randomised controlled trial. Lancet. 2010; 376: 1989-1999.

22. Berenson JR. New advances in the biology and treatment of myeloma bone disease. Semin Hematol. 2001; 38: 15-20.

23. Rosen LS, Gordon D, Kaminski M, Howell A, Belch A, Mackey J, Apffelstaedt J, Hussein M, Coleman RE, Reitsma DJ, Seaman JJ, Chen BL and Ambros Y. Zoledronic acid versus pamidronate in the treatment of skeletal metastases in patients with breast cancer or osteolytic lesions of multiple myeloma: a phase III, double-blind, comparative trial. Cancer J. 2001; 7: 377-387.

24. Barrett-Lee P, Casbard A, Abraham J, Hood K, Coleman R, Simmonds P, Timmins H, Wheatley D, Grieve R, Griffiths $\mathrm{G}$ and Murray N. Oral ibandronic acid versus intravenous zoledronic acid in treatment of bone metastases from breast cancer: a randomised, open label, non-inferiority phase 3 trial. Lancet Oncol. 2014; 15: 114-122. 
25. Coleman R, Cameron D, Dodwell D, Bell R, Wilson C, Rathbone E, Keane M, Gil M, Burkinshaw R, Grieve R, Barrett-Lee P, Ritchie D, Liversedge V, et al. Adjuvant zoledronic acid in patients with early breast cancer: final efficacy analysis of the AZURE (BIG 01/04) randomised open-label phase 3 trial. Lancet Oncol. 2014; 15: 997-1006.

26. Gnant M, Mlineritsch B, Stoeger H, Luschin-Ebengreuth G, Heck D, Menzel C, Jakesz R, Seifert M, Hubalek M, Pristauz G, Bauernhofer T, Eidtmann H, Eiermann W, et al. Adjuvant endocrine therapy plus zoledronic acid in premenopausal women with early-stage breast cancer: 62-month follow-up from the ABCSG-12 randomised trial. Lancet Oncol. 2011; 12: 631-641.

27. Caraglia M, Santini D, Marra M, Vincenzi B, Tonini G, Budillon A. Emerging anti-cancer molecular mechanisms of aminobisphosphonates. Endocr Relat Cancer. 2006; 13: 7-26.

28. Berridge MJ, Bootman MD, Roderick HL. Calcium signalling: dynamics, homeostasis and remodelling. Nat Rev Mol Cell Biol. 2003; 4: 517-529.

29. Noren DP, Chou WH, Lee SH, Qutub AA, Warmflash A, Wagner DS, Popel AS and Levchenko A. Endothelial cells decode VEGF-mediated $\mathrm{Ca} 2+$ signaling patterns to produce distinct functional responses. Sci Signal. 2016; 9: ra20.

30. Jia XH, Du Y, Mao D, Wang ZL, He ZQ, Qiu JD, Ma XB, Shang WT, Ding D and Tian J. Zoledronic acid prevents the tumor-promoting effects of mesenchymal stem cells via MCP-1 dependent recruitment of macrophages. Oncotarget. 2015; 6: 26018-26028. doi: 10.18632/oncotarget.4658.

31. Senaratne SG, Pirianov G, Mansi JL, Arnett TR, Colston $\mathrm{KW}$. Bisphosphonates induce apoptosis in human breast cancer cell lines. Br J Cancer. 2000; 82: 1459-1468.

32. Tassone P, Tagliaferri P, Viscomi C, Palmieri C, Caraglia M, D'Alessandro A, Galea E, Goel A, Abbruzzese A, Boland $\mathrm{CR}$ and Venuta S. Zoledronic acid induces antiproliferative and apoptotic effects in human pancreatic cancer cells in vitro. Br J Cancer. 2003; 88: 1971-1978.

33. Coleman RE, Marshall H, Cameron D, Dodwell D, Burkinshaw R, Keane M, Gil M, Houston SJ, Grieve RJ, Barrett-Lee PJ, Ritchie D, Pugh J, Gaunt C, et al. Breastcancer adjuvant therapy with zoledronic acid. N Engl J Med. 2011; 365: 1396-1405.

34. Facchini G, Caraglia M, Morabito A, Marra M, Piccirillo MC, Bochicchio AM, Striano S, Marra L, Nasti G, Ferrari
E, Leopardo D, Vitale G, Gentilini D, et al. Metronomic administration of zoledronic acid and taxotere combination in castration resistant prostate cancer patients: phase I ZANTE trial. Cancer Biol Ther. 2010; 10: 543-548.

35. Chiarugi P, Paoli P, Cirri P. Tumor microenvironment and metabolism in prostate cancer. Semin Oncol. 2014; 41: 267-280.

36. Josson S, Matsuoka Y, Chung LW, Zhau HE, Wang R. Tumor-stroma co-evolution in prostate cancer progression and metastasis. Semin Cell Dev Biol. 2010; 21: 26-32.

37. Rietkotter E, Menck K, Bleckmann A, Farhat K, Schaffrinski M, Schulz M, Hanisch UK, Binder C and Pukrop T. Zoledronic acid inhibits macrophage/microgliaassisted breast cancer cell invasion. Oncotarget. 2013; 4: 1449-1460. doi: 10.18632/oncotarget.1201.

38. Galdiero MR, Garlanda C, Jaillon S, Marone G, Mantovani A. Tumor associated macrophages and neutrophils in tumor progression. J Cell Physiol 2013; 228: 1404-1412.

39. Clezardin P. Anti-tumour activity of zoledronic acid. Cancer Treat Rev. 2005; 31: 1-8.

40. Meyer-Ter-Vehn T, Katzenberger B, Han H, Grehn F, Schlunck G. Lovastatin inhibits TGF-beta-induced myofibroblast transdifferentiation in human tenon fibroblasts. Invest Ophthalmol Vis Sci. 2008; 49: 3955-3960.

41. Mitra AK, Zillhardt M, Hua Y, Tiwari P, Murmann AE, Peter ME and Lengyel E. MicroRNAs reprogram normal fibroblasts into cancer-associated fibroblasts in ovarian cancer. Cancer Discov. 2012; 2: 1100-1108.

42. Madsen CD, Pedersen JT, Venning FA, Singh LB, Moeendarbary E, Charras G, Cox TR, Sahai E and Erler JT. Hypoxia and loss of PHD2 inactivate stromal fibroblasts to decrease tumour stiffness and metastasis. EMBO Rep. 2015; 16: 1394-1408.

43. Margheri F, Chilla A, Laurenzana A, Serrati S, Mazzanti B, Saccardi R, Santosuosso M, Danza G, Sturli N, Rosati F, Magnelli L, Papucci L, Calorini L, et al. Endothelial progenitor cell-dependent angiogenesis requires localization of the full-length form of uPAR in caveolae. Blood. 2011; 118: 3743-3755.

44. Yoder MC. Developing reagents and conditions to induce mesoderm subsets from ES cells. Cell Stem Cell. 2007; 1: 603-604. 